DOI https://doi.org/10.24297/jam.v19i.8885

\title{
On the solvability of a nonlinear functional integral equations via measure of noncompactness in $L^{p}\left(\mathbb{R}^{N}\right)$
}

\author{
Wagdy G. El-Sayed ${ }^{1}$, Mahmoud M. El-Borai ${ }^{2}$, Mohamed M.A. Metwali $^{3}$, Nagwa I. Shemais ${ }^{4}$ \\ ${ }^{1,2}$ Faculty of Science, Alexandria University, Alexandria, Egypt \\ ${ }^{3,4}$ Department of mathematics, Faculty of Science, Damanhour Universty, Egypt \\ ${ }^{1}$ wagdygoma@alexu.edu.eg, ${ }^{2}$ m_m_elborai@yahoo.com, ${ }^{3}$ m.metwali@yahoo.com, ${ }^{4}$ n.ibrahim90@yahoo.com
}

\begin{abstract}
Using the technique of a suitable measure of non-compactness and the Darbo fixed point theorem, we investigate the existence of a nonlinear functional integral equation of Urysohn type in the space of Lebesgue integrable functions $L^{p}\left(\mathbb{R}^{N}\right)$. In this space, we show that our functional-integral equation has at least one solution. Finally an example is also discussed to indicate the natural realizations of our abstract result.
\end{abstract}

Keywords: functional integral equation; measure of noncompactnes; existence; Darbo's fixed point theorem; fixed point.

Biographical notes: Wagdy G. El-Sayed received his PhD in Integral Equations from Maria Curie - Sklodowiska University in Lublin - Poland. He is currently an Associate Professor and Professor at the Department Mathematics and Computer Science, Faculty of Science, Alexandria University, Alexandria - Egypt. His research is interesting in the Solvability of some different integral equations or functional integral equations or integro-differential equations or quadratic integral equations in different classes of integrable functions, where he try to assume the sufficient conditions under which the existence theorem of a certain integral equation can be proved by using Darbo fixed point theorem via some measures of noncompactness. He published in journals such as Journal of Mathematical Analysis and Applications, Budapest University, Hungary, International Journal of Mathematics, Florida - USA, Journal of Applied Mathematics and Computation

Mahmoud M. El-Borai Editor in this Journal

Mohamed M.A. Metwali obtained his Ph.D. from A. Mickiewicz University, Poznan, Poland in topic of "Differential and integral equations". He is currently working as Associate Professor at Department of Mathematics, Faculty of Science, Damnhour University, Egypt.

His research interests are in the areas of Integral Equations, Differential Equations, Function Analysis, set valued Analysis, time scales, fixed point theory, and solutions in different function spaces such as "Lesbegue spaces, Orlicz spaces, space of regulated functions, space of Bounded variation, space of continuous functions". He published many papers in various journals such as J. Math. Anal. Appl., Mediter. J. Math, Electron. J. Qual. Theory of Differ. Equ., Opuscula Math., Math. Slovaca, J. Fixed Point Theory Appl., Carpathian J. Math., Rocky Mountain J. Math., and Demonstratio Mathematica.

Nagwa I. Shemais received her MSc in Dynamical system. She is currently a PhD candidate at the Department of Mathematics, Faculty of Science, Damanhour University, Egypt. Her research interests are in the areas of nonlinear analysis. 


\section{Introduction}

Integral equations appear in many applications in describing numerous real world problems (see, for instance, ([30], [31], [5], [18]), and references therein).

Also many useful applications in describing problems of the real world and numerous events, which appear in physics, engineering, mechanics, biology, etc. See for example [1, 4, 8, 13, 15] can be depicted and demonstrated by methods of non-linear functional integral equations (for example, we refer to [25, 26, 28]). Apart from that, integral equations are often investigated in research papers and monographs (cf. [6, 12, 16, 18, 29, 32]) and the references cited therein.

\section{Preliminaries}

We will collect in this section some definitions and basic results which will be used further on throughout the paper. First, we denote $L^{p}(U)\left(U \in \mathbb{R}^{N}\right)$ as the space of Lebesgue integrable functions on $U$ with the standard norm $\|x\|_{L^{p}(U)}=\left(\int_{U}\right.$ $|x(t)|^{p} d t^{\frac{1}{p}}$.

\section{Theorem 2.1 [1, 8, 9]}

Let $F$ be a bounded set in $L^{p}\left(\mathbb{R}^{N}\right)$ with $1 \leq p<\infty$. The closure of $F$ in $L^{p}\left(\mathbb{R}^{N}\right)$ is compact if and only if

$$
\lim _{h \rightarrow 0}\left\|\tau_{h} f-f\right\|_{L^{p}\left(\mathbb{R}^{N}\right)}=0 \quad \text { uniformly in } f \in F,
$$

where $\tau_{h} f(x)=f(x+h)$ for all $x, h \in \mathbb{R}^{N}$. Also for $\epsilon>0$ there is a bounded and measurable subset $\Omega \subset\left(\mathbb{R}^{N}\right)$ such that

$$
\|f\|_{\left(\mathbb{R}^{N} \backslash \Omega\right)}<\epsilon \quad \text { forall } f \in F \text {. }
$$

Next, we recall the concept of measure of noncompactness, let $E$ be an infinite dimensional Banach space with norm $\|$.$\| and zero element \theta$. Denote by $\mathcal{M}_{E}$ the family of all nonempty and bounded subsets of $E, \mathcal{N}_{E}$ and $\mathcal{N}_{E}^{W}$

the family of all nonempty relatively compact

and weakly relatively compact sets, respectively. The symbols $\bar{X}$ and ConvX stand for the closure and closed convex hull of a subset $X$ of $E$, respectively. We use the standard notation $X+Y$ and $\lambda X$ for algebraic operations on sets, while,

we denote $B_{r}=B(\theta, r)$ the closed ball centered at $\theta$ and with radius $r$.

Definition 2.1 (Measure of noncompactness)

[12]

A mapping $\mu: \mathcal{M}_{E} \rightarrow[0, \infty)$ is said to be a measure of noncompactness in $E$ if it satisfies the following conditions: 
(1) the family $\operatorname{ker} \mu=\left\{X \in \mathcal{M}_{E}: \mu(X)=0\right\}$ is nonempty and ker $\mu \subset \mathcal{N}_{E}$, where ker $\mu$ is called the kernel of the measure $\mu$.

(2) $X \subset Y \Rightarrow \mu(X) \leq \mu(Y)$.

(3) $\mu(\operatorname{Conv} X)=\mu(X)=\mu(\bar{X})$.

(4) $\mu[\lambda X+(1-\lambda) Y] \leq \lambda \mu(X)+(1-\lambda) \mu(Y), \lambda \in[0,1]$.

(5) If $X_{n} \in \mathcal{M}_{E}, X_{n}=\bar{X}_{n}$ and

$X_{n+1} \subset X_{n}$ for $n=1,2, \ldots$ and if

$\lim _{n \rightarrow \infty} \mu\left(X_{n}\right)=0$, then

$X_{\infty}=\bigcap_{n=1}^{\infty} X_{n} \neq \phi$.

\section{Theorem 2.2 [1]}

Suppose $1 \leq p<\infty$ and $X$ is

a bounded subset of $\left(\mathbb{R}^{N}\right)$. For $x \in X$ and $\epsilon>0$

$w^{T}(x, \epsilon)=\sup \left\{\left\|\tau_{h} x-x\right\|_{L^{p}\left(B_{T}\right)}:\|h\|_{\mathbb{R}^{N}}<\epsilon\right\}$,

$w^{T}(X, \epsilon)=\sup \left\{w^{T}(x, \epsilon): x \in X\right\}$,

$w^{T}(X)=\lim _{\epsilon \rightarrow 0} w^{T}(X, \epsilon)$,

$w(X)=\lim _{T \rightarrow \infty} w^{T}(X)$

$d(X)=\lim _{T \rightarrow \infty} \sup \left\{\|x\|_{L^{p}\left(\mathbb{R}^{N} \backslash B_{T}\right)}: x \in X\right\}$,

where $B_{T}=\left\{a \in \mathbb{R}^{\mathbb{N}}:\|a\|_{\mathbb{R}^{\mathbb{N}}} \leq T\right\}$. Then

$$
\mu(X)=w(X)+d(X)
$$

is a measure of non compactness on $L^{p}\left(\mathbb{R}^{N}\right)$.

At the end of this section, we recall the fixed point theorem due to Darbo which enables us to prove the existence theorem for solutions of a several integral equations considered in nonlinear analysis. To quote this theorem we need the following definitions. 
Definition 2.2 [12]

The function $f: I \times \mathbb{R} \rightarrow \mathbb{R}$ satisfies Carathéodory condition if it satisfies the following two conditions:

(1) $f$ is measurable in $t \in I$ for any $x \in \mathbb{R}$.

(2) $f$ is continuous in $x \in \mathbb{R}$ for almost all $t \in I$.

Definition 2.3 (Darbo condition) [11]

Let $\Omega$ be a nonempty subset of a Banach space $E$ and let $A: \Omega \rightarrow E$ be a continuous operator which transforms bounded sets onto bounded ones. We say that $A$ satisfies the Darbo condition (with a constant $k \geq 0$ ) with respect to a measure of noncompactness $\mu$ if for any bounded subset $X$ of

$\Omega$, we have $\mu(A X) \leq k \mu(X)$.

Note that, if $A$ satisfies the Darbo condition with $k<1$, then it is called a contraction operator with respect to $\mu$.

Theorem 2.3 (Darbo fixed point theorem) [7]

Let $\Omega$ be a nonempty, bounded, closed and convex subset of $E$ and let $f: \Omega \rightarrow \Omega$ be a continuous transformation which is a contraction with respect to the measure of noncompactness $\mu$, i.e. there exists a constant $k \in[0,1)$ such that $\mu(f X) \leq k \mu(X)$

for any nonempty subset $X$ of $\Omega$. Then $f$ has at least one fixed point in the set $\Omega$.

\section{Main result}

This section is devoted to discuss the solvability of the following nonlinear functional integral equation

$$
u(x)=f(x)+g_{1}(x, u(x))+h_{1}\left(x, g_{2}(x, u(x)), \int_{\mathbb{R}^{N}} h_{2}(x, y,(Q u)(y)) d y\right)
$$

Now, we will try to assume some assumptions under which we can prove our existence theorem.

Assume the following conditions are satisfied:

(i) $f \in L^{p}\left(\mathbb{R}^{N}\right)$;

(ii) $g_{i}: \mathbb{R}^{N} \times \mathbb{R} \rightarrow \mathbb{R}$ satisfy Carathéodory condition

(i.e. measurable in $t$ for all $x \in \mathbb{R}^{N}$, and continuous in $x$ for all $t \in \mathbb{R})$ and there exists a constant $l \in[0,1)$ and $a_{i} \in L^{p}\left(\mathbb{R}^{N}\right)$

such that

$$
\left|g_{i}(x, u)-g_{i}(y, v)\right| \leq\left|a_{i}(x)-a_{i}(y)\right|+l|u-v|,
$$

for any $u, v \in \mathbb{R}$ and almost all $x, y \in \mathbb{R}^{N}$ where $i=1,2$. 
(iii) $h_{1}: \mathbb{R}^{N} \times \mathbb{R} \times \mathbb{R} \rightarrow \mathbb{R}$ such that

$$
\left|h_{1}(x, y, z)\right| \leq a(x, y)+b_{1}|z|
$$

for all $x, y \in \mathbb{R}^{N}, a \in L^{q}\left(\mathbb{R}^{N}\right)$, where $|a(x, y)| \leq a_{3}(x)+b_{2}|y|$ where $b_{1}, b_{2} \geq 0$ are constant and $a_{3} \in L^{q}\left(\mathbb{R}^{N}\right)$.

(iv) $\left|h_{2}(x, y, u)\right| \leq k(x, y)\left\{a_{4}(y)+b|u|\right\}$, where $h_{2}: \mathbb{R}^{N} \times \mathbb{R}^{N} \times \mathbb{R} \rightarrow \mathbb{R}, b>0, a_{4} \in L^{p}\left(\mathbb{R}^{N}\right)$ and $k(x, y)$ satisfies Carathéodory condition $k: \mathbb{R}^{N} \times \mathbb{R}^{N} \rightarrow \mathbb{R}$ and there exist $f$

$1, f_{2} \in L^{p}\left(\mathbb{R}^{N}\right)$ and $f^{*} \in L^{q}\left(\mathbb{R}^{N}\right)\left(\frac{1}{p}+\frac{1}{q}=1\right)$ such that $|k(x, y)| \leq f^{*}(y) f_{1}(x)$, for all $x, y \in \mathbb{R}^{N}$ and

$$
\left|k\left(x_{1}, y\right)-k\left(x_{2}, y\right)\right| \leq f^{*}(y)\left|f_{2}\left(x_{1}\right)-f_{2}\left(x_{2}\right)\right| .
$$

(v) The operator $Q$ is bounded linear operator and continuously maps the space $L^{p}\left(\mathbb{R}^{N}\right)$ into itself. Moreover, there exists a nondecreasing function $\psi: \mathbb{R}_{+} \rightarrow \mathbb{R}_{+}$such that

$$
\|Q u\|_{L^{p}\left(\mathbb{R}^{N}\right)} \leq \psi\left(\|u\|_{L^{p}\left(\mathbb{R}^{N}\right)}\right)
$$

for any $u \in L^{p}\left(\mathbb{R}^{N}\right)$.

(vi) there exists a positive constant $r_{0}$ such that

$$
\begin{aligned}
& \|\quad f\|_{L^{p}\left(\mathbb{R}^{N}\right)}+l r_{0}+\left\|g_{1}(x, 0)\right\|_{L^{p}\left(\mathbb{R}^{N}\right)}+\left\|a_{3}\right\|_{L^{p}\left(\mathbb{R}^{N}\right)}+b_{2} l r_{0} \\
& +\quad b_{2}\left\|g_{2}(x, 0)\right\|_{L^{p}\left(\mathbb{R}^{N}\right)}+b_{1}\|K\|_{1}\left\|a_{4}\right\|_{L^{p}\left(\mathbb{R}^{N}\right)}+b b_{1}\|K\|_{1} \psi\left(r_{0}\right)
\end{aligned}
$$

$\leq r_{0}$, where

$$
(K u)(t)=\int_{\mathbb{R}^{N}} k(x, y) u(y) d y
$$

and

$$
\|K\|_{1}=\left\{S u p\|K u\|_{L^{p}\left(\mathbb{R}^{N}\right)} \quad:\|u\| \leq r\right.
$$

$0\}$.

Remark 3.1 The linear fredholm integral operator $K: L^{p}\left(\mathbb{R}^{N}\right) \rightarrow L^{p}\left(\mathbb{R}^{N}\right)$ is a continuous operator and $\|K\|_{1} \leq \infty$.

Theorem 3.1 If the above assumptions (i)-(vi) are satisfied then the functional integral equation 1 has at least one solution in $L^{p}\left(\mathbb{R}^{N}\right)$.

Proof: First of all, we define the operator $F: L^{p}\left(\mathbb{R}^{N}\right) \rightarrow L^{p}\left(\mathbb{R}^{N}\right)$ by

$$
(F u)(x)=f(x)+g_{1}(x, u(x))+h_{1}\left(x, g_{2}(x, u(x)), \int_{\mathbb{R}^{N}} h_{2}(x, y,(Q u)(y)) d y\right),
$$

and $(G U)(x)=h_{1}\left(x, g_{2}(x, u(x)), \int_{\mathbb{R}^{N}} h_{2}(x, y,(Q u)(y)) d y\right)$. Now Fu is measurable for anyu $\in L^{p}\left(\mathbb{R}^{N}\right)$, we will prove that $F u \in L^{p}\left(\mathbb{R}^{N}\right)$ for any $u \in L^{p}\left(\mathbb{R}^{N}\right)$ as $G: L^{p}\left(\mathbb{R}^{N}\right) \rightarrow L^{p}\left(\mathbb{R}^{N}\right)$ using the above conditions, we have the following inequality

$|(G u)(x)|=\left|h_{1}\left(x, g_{2}(x, u(x)), \int_{\mathbb{R}^{N}} h_{2}(x, y,(Q u)(y)) d y\right)\right|$ 


$$
\begin{aligned}
& \leq a\left(x, g_{2}(x, u(x))\right)+b_{1}\left|\int_{\mathbb{R}^{N}} h_{2}(x, y,(Q u)(y)) d y\right| \\
& \leq a_{3}(x)+b_{2}\left|g_{2}(x, u(x))\right|+b_{1} \int_{\mathbb{R}^{N}}\left|h_{2}(x, y,(Q u)(y))\right| d y \\
& \leq a_{3}(x)+b_{2}\left|g_{2}(x, u(x))-g_{2}(x, 0)\right|+b_{2}\left|g_{2}(x, 0)\right| \\
& +b_{1} \int_{\mathbb{R}^{N}} k(x, y)\left[a_{4}(y)+b|(Q u)(y)|\right] d y \\
& \leq a_{3}(x)+b_{2}\left|a_{2}(x)-a_{2}(x)\right|+b_{2} l|u|+b_{2}\left|g_{2}(x, 0)\right| \\
& +b_{1} \int_{\mathbb{R}^{N}} k(x, y) a_{4}(y) d y+b b_{1} \int_{\mathbb{R}^{N}} k(x, y)|(Q u)(y)| d y \\
& \leq a_{3}(x)+b_{2} l|u|+b_{2}\left|g_{2}(x, 0)\right|+b_{1} \int_{\mathbb{R}^{N}} k(x, y) a_{4}(y) d y \\
& +b b_{1} \int_{\mathbb{R}^{N}} k(x, y)|(Q u)(y)| d y, \\
& \|G u\|_{L^{p}\left(\mathbb{R}^{N}\right)} \leq\left\|a_{3}\right\|_{L^{p}\left(\mathbb{R}^{N}\right)}+b_{2} l\|u\|_{L^{p}\left(\mathbb{R}^{N}\right)}+b_{2}\left\|g_{2}(x, 0)\right\|_{L^{p}\left(\mathbb{R}^{N}\right)} \\
& +b_{1}\|K\|_{1}\left\|a_{4}\right\|_{L^{p}\left(\mathbb{R}^{N}\right)}+b b_{1}\|K\|_{1}\|Q u\|_{L^{p}\left(\mathbb{R}^{N}\right)} \\
& <\infty,
\end{aligned}
$$

then from assumptions(i), (ii), $F(u) \in L^{p}\left(\mathbb{R}^{N}\right)$ and $F$ is will defined

$$
\begin{aligned}
& |(F u)(x)| \leq|f(x)|+ \\
& \left|g_{1}(x, u(x))\right|+|G x| \\
& \leq|f(x)|+l|u|+\left|g_{1}(x, 0)\right|+|G x| \\
& \|F u\|_{L^{p}\left(\mathbb{R}^{N}\right)} \leq\|f\|_{L^{p}\left(\mathbb{R}^{N}\right)}+l\|u\|_{L^{p}\left(\mathbb{R}^{N}\right)}+\left\|g_{1}(x, 0)\right\|_{L^{p}\left(\mathbb{R}^{N}\right)}+\|G\|_{L^{p}\left(\mathbb{R}^{N}\right)} \\
& \leq\|f\|_{L^{p}\left(\mathbb{R}^{N}\right)}+l\|u\|_{L^{p}\left(\mathbb{R}^{N}\right)}+\left\|g_{1}(x, 0)\right\|_{L^{p}\left(\mathbb{R}^{N}\right)}+\left\|a_{3}\right\|_{L^{p}\left(\mathbb{R}^{N}\right)} \\
& +b_{2} l\|u\|_{L^{p}\left(\mathbb{R}^{N}\right)}+b_{2}\left\|g_{2}(x, 0)\right\|_{L^{p}\left(\mathbb{R}^{N}\right)} \\
& +b_{1}\|K\|_{1}\left\|a_{4}\right\|_{L^{p}\left(\mathbb{R}^{N}\right)}+b b_{1}\|K\|_{1}\|Q u\|_{L^{p}\left(\mathbb{R}^{N}\right)} \\
& <\infty \text {. }
\end{aligned}
$$

Next, we show that

$$
F: B_{r_{0}} \rightarrow B_{r_{0}} \text { where }
$$


$B_{r_{0}}$ is closed ball of radius $r_{0}$ is constant, let $u \in B_{r_{0}}$ where $\left(\|u\| \leq r_{0}\right)$

$$
\begin{aligned}
\|F u\|_{L^{p}\left(\mathbb{R}^{N}\right)} & \leq\|f\|_{L^{p}\left(\mathbb{R}^{N}\right)}+l r_{0}+\left\|g_{1}(x, 0)\right\|_{L^{p}\left(\mathbb{R}^{N}\right)}+\left\|a_{3}\right\|_{L^{p}\left(\mathbb{R}^{N}\right)}+b_{2} l r_{0} \\
& +b_{2}\left\|g_{2}(x, 0)\right\|_{L^{p}\left(\mathbb{R}^{N}\right)}+b_{1}\|K\|_{1}\left\|a_{4}\right\|_{L^{p}\left(\mathbb{R}^{N}\right)} \\
& +b b_{1}\|K\|_{1} \psi\left(r_{0}\right)
\end{aligned}
$$

$\leq r_{0}$

Now, we show that $w_{0}(F X) \leq l\left(b_{2}+1\right) w_{0}(X)$ for any nonempty set $X \subset B_{r_{0}}$. To do this, we fix arbitrary $T>0$ and $\epsilon>0$, let us choose $u \in X$ and for $x, h \in B_{T}$ with $\|h\|_{\mathbb{R}^{N}} \leq \epsilon$, we have

$$
\begin{aligned}
& /(G u)(x+h)-(G u)(x) / \\
& =\mid h_{1}\left(x+h, g_{2}(x+h, u(x+h)), \int_{\mathbb{R}^{N}} h_{2}(x+h, y,(Q u)(y)) d y\right) \\
& \text { - } h_{1}\left(x, g_{2}(x, u(x)), \int_{\mathbb{R}^{N}} h_{2}(x, y,(Q u)(y)) d y\right) \\
& \leq\left|a_{3}(x+h)+b_{2}\right| g_{2}(x+h, u(x+h))\left|-a_{3}(x)-b_{2}\right| g_{2}(x, u(x))|| \\
& +b_{1}\left(\left|\int_{\mathbb{R}^{N}} h_{2}(x+h, y,(Q u)(y)) d y\right|-\left|\int_{\mathbb{R}^{N}} h_{2}(x, y,(Q u)(y)) d y\right|\right) \\
& \leq\left|a_{3}(x+h)-a_{3}(x)\right|+b_{2}\left|g_{2}(x+h, u(x+h))-g_{2}(x, u(x))\right| \\
& +b_{1}\left(\int_{\mathbb{R}^{N}} k(x+h, y)\left[a_{4}(y)+b|(Q u)(y)|\right] d y\right. \\
& \text { - } \int_{\mathbb{R}^{N}} k(x, y) \\
& \left.\times\left[a_{4}(y)+b|(Q u)(y)|\right] d y\right) \\
& \leq\left|a_{3}(x+h)-a_{3}(x)\right|+b_{2}\left|g_{2}(x+h, u(x+h))-g_{2}(x, u(x))\right| \\
& +b_{1}\left(\int_{\mathbb{R}^{N}}|k(x+h, y)-k(x, y)|\left[a_{4}(y)+b|(Q u)(y)|\right] d y\right) \\
& \leq\left|a_{3}(x+h)-a_{3}(x)\right|+b_{2}\left|g_{2}(x+h, u(x+h))-g_{2}(x+h, u(x))\right| \\
& +b_{2}\left|g_{2}(x+h, u(x))-g_{2}(x, u(x))\right|+b_{1} \int_{\mathbb{R}^{N}} f^{*}(y)\left(\left|f_{2}(x+h)-f_{2}(x)\right|\right) \\
& \times\left[a_{4}(y)+b|(Q u)(y)|\right] d y \\
& \leq\left|a_{3}(x+h)-a_{3}(x)\right|+b_{2} l|u(x+h)-u(x)|+b_{2}\left(\left|a_{2}(x+h)-a_{2}(x)\right|\right. \\
& \left.+b_{2} l|u(x)-u(x)|\right)+b_{1} \int_{\mathbb{R}^{N}} f^{*}(y)\left|f_{2}(x+h)-f_{2}(x)\right| a_{4}(y) d y \\
& +b b_{1} \int_{\mathbb{R}^{N}} f^{*}(y)\left|f_{2}(x+h)-f_{2}(x) \|(Q u)(y)\right| d y .
\end{aligned}
$$




$$
\left\|\tau_{h} G u-G u\right\|_{L^{p}}=\left(\int_{B^{T}}|(G u)(x+h)-(G u)(x)|^{p} d x\right)^{\frac{1}{p}}
$$

$\leq\left(\int_{B^{T}}\left|a_{3}(x+h)-a_{3}(x)\right|^{p} d x\right)^{\frac{1}{p}}+l b_{2}\left(\int_{B^{T}}|u(x+h)-u(x)|^{p} d x\right)^{\frac{1}{p}}$

$+\left(\int_{B^{T}} b_{2}\left|a_{2}(x+h)-a_{2}(x)\right|^{p} d x\right)^{\frac{1}{p}}$

$+b_{1}$

$\left.\left[\int_{B^{T}}\left(\int_{\mathbb{R}^{N}}\left|f^{*}(y)\right|^{q} a_{4}(y)\left|f_{2}(x+h)-f_{2}(x)\right|^{q}\right)\left|a_{2}(y)\right|^{q} d y\right)^{\frac{p}{q}} d x\right]^{\frac{1}{p}}$

$$
+b b_{1}\left[\int_{B^{T}}\left(\int_{\mathbb{R}^{N}}\left|f^{*}(y)\right|^{q}\left|f_{2}(x+h)-f_{2}(x)\right|^{q}|(Q u)(y)|^{q} d y\right)^{\frac{p}{q}} d x\right]^{\frac{1}{p}}
$$

$\left\|\tau_{h} G u-G u\right\|_{L^{p}}$

$\leq\left\|\tau_{h} a_{3}-a_{3}\right\|_{L^{p}\left(B^{T}\right)}+l b_{2}\left\|\tau_{h} u-u\right\|_{L^{p}\left(B^{T}\right)}+b_{2}\left\|\tau_{h} a_{2}-a_{2}\right\|_{L^{p}\left(B^{T}\right)}$

$+b_{1}\left\|f^{*}\right\|_{L^{q}\left(\mathbb{R}^{N}\right)}$

$\times\left\|\tau_{h} f_{2}-f_{2}\right\|_{L^{p}\left(B^{T}\right)}\left\|a_{4}\right\|_{L^{p}\left(\mathbb{R}^{N}\right)}$

$+b b_{1}\left\|f^{*}\right\|$

$\left.L^{q} \mathbb{R}^{N}\right)\left\|\tau_{h} f_{2}-f_{2}\right\|_{L^{p}\left(B^{T}\right)}\|Q u\|_{L^{p}\left(\mathbb{R}^{N}\right)}$

$\leq w^{T}\left(a_{3}, \epsilon\right)+l b_{2} w^{T}(u, \epsilon)+b_{2} w^{T}\left(a_{2}, \epsilon\right)$

$+b_{1} w^{T}\left(f_{2}, \epsilon\right)\left\|f^{*}\right\|_{L^{q}\left(\mathbb{R}^{N}\right)}\left\|a_{4}\right\|_{L^{p}\left(\mathbb{R}^{N}\right)}+b b_{1}\left\|f^{*}\right\|_{L^{q}\left(\mathbb{R}^{N}\right)}$

$w^{T}\left(f_{2}, \epsilon\right) \psi(\|u\|)_{L^{p}\left(\mathbb{R}^{N}\right)}$.

$|(F u)(x+h)-(F u)(x)|$ 
$\leq|f(x+h)-f(x)|+\left|g_{1}(x+h, u(x+h))-g_{1}(x, u(x))\right|$

$+|(G u)(x+h)-(G u)(x)|$

$\leq|f(x+h)-f(x)|+\left|g_{1}(x+h, u(x+h))-g_{1}(x+h, u(x))\right|$

$+\left|g_{1}(x+h, u(x))-g(x, u(x))\right|+|(G u)(x+h)-(G u)(x)|$

$\leq|f(x+h)-f(x)|+\left|a_{1}(x+h)-a_{1}(x)\right|+l|u(x+h)-u(x)|$

$+|(G u)(x+h)-(G u)(x)|$

$\left\|\tau_{h} F u-F u\right\|_{L^{p}} \leq\left(\int_{B^{T}}|f(x+h)-f(x)|^{p} d x\right)^{\frac{1}{p}}+l\left(\int_{B^{T}}|u(x+h)-u(x)|^{p} d x\right)^{\frac{1}{p}}$

$+$

$\left(\int_{B^{T}}\left|a_{1}(x+h)-a_{1}(x)\right|^{p} d x\right)^{\frac{1}{p}}+\left\|\tau_{h} G u-G u\right\|_{\left.L^{p}\left(B^{T}\right)\right)}$

$\leq\left\|\tau_{h} f-f\right\|_{L^{p}\left(B^{T}\right)}+l\left\|\tau_{h} u-u\right\|_{L_{p}\left(B^{T}\right)}+\mid \tau_{h} a_{1}-a_{1} \|_{L^{p}\left(B^{T}\right)}$

$+\left\|\tau_{h} G-G\right\|_{L^{p}\left(B^{T}\right)}$

$$
\begin{aligned}
w^{T}(F x, \epsilon) & \leq w^{T}(f, \epsilon)+l w^{T}(u, \epsilon)+w^{T}\left(a_{1}, \epsilon\right)+w^{T}\left(a_{3}, \epsilon\right)+l b_{2} w^{T}(u, \epsilon) \\
& +w^{T}\left(a_{2}, \epsilon\right)+b_{1} w^{T}\left(f_{2}, \epsilon\right)\left\|f^{*}\right\|_{L_{q}\left(\mathbb{R}^{N}\right)}\left\|a_{4}\right\|_{L_{p}\left(\mathbb{R}^{N}\right)} \\
& +b b_{1}\left\|f^{*}\right\|_{L_{q}\left(\mathbb{R}^{N}\right)}
\end{aligned}
$$

$w^{T}\left(f_{2}, \epsilon\right) \psi(\|u\|)_{L_{p}\left(\mathbb{R}^{N}\right)}$

Thus, we obtain

$$
\begin{aligned}
w^{T}(F X, \epsilon) & \leq w^{T}(f, \epsilon)+l w^{T}(X, \epsilon)+w^{T}\left(a_{1}, \epsilon\right)+w^{T}\left(a_{3}, \epsilon\right)+l b_{2} w^{T}(u, \epsilon) \\
& +w^{T}\left(a_{2}, \epsilon\right)+b_{1} w^{T}\left(f_{2}, \epsilon\right)\left\|f^{*}\right\|_{L_{q}\left(\mathbb{R}^{N}\right)}\left\|a_{4}\right\|_{L_{p}\left(\mathbb{R}^{N}\right)} \\
& +b b_{1}\left\|f^{*}\right\|_{L_{q}\left(\mathbb{R}^{N}\right)}
\end{aligned}
$$

$w^{T}\left(f_{2}, \epsilon\right) \psi\left(r_{0}\right)$

Also, we have $w^{T}\left(f_{2}, \epsilon\right), w^{T}(f, \epsilon)$, and $w^{T}\left(a_{i}, \epsilon\right) \rightarrow 0$ as $\epsilon \rightarrow \infty$ where $i=1,2,3$

then, we obtain

$$
w(F X) \leq l\left(b_{2}+1\right) w(X), \quad \text { where } l\left(b_{2}+1\right) \leq 1
$$


Next, let us fix an arbitrary number $T>0$, then taking into account our assumptions,

for an arbitrary function $u \in X$. We have

$\left(\int_{\mathbb{R}^{N}}\right.$

$\left.\backslash B^{T}|(F u)(x)|^{p} d x\right)^{\frac{1}{p}} \leq\left(\int_{\mathbb{R}^{N} \backslash B^{T}}|f(x)|^{p} d x\right)^{\frac{1}{p}}+\left(\int_{\mathbb{R}^{N} \backslash B^{T}}\left|g_{1}(x, u(x))\right|^{p} d x\right)^{\frac{1}{p}}$

$+\left(\int_{\mathbb{R}^{N} \backslash B^{T}}\left|h_{1}\left(x, g_{2}(x, u(x)), \int_{\mathbb{R}^{N}} h_{2}(x, y,(Q u)(y)) d y\right)\right|^{p} d x\right)^{\frac{1}{p}}$

$\leq\left(\int_{\mathbb{R}^{N} \backslash B^{T}}|f(x)|^{p} d x\right)^{\frac{1}{p}}+\left(\int_{\mathbb{R}^{N} \backslash B^{T}}\left|g_{1}(x, u(x))-g_{1}(x, 0)\right|^{p} d x\right)^{\frac{1}{p}}$

$+\left(\int_{\mathbb{R}^{N} \backslash B^{T}}\left|g_{1}(x, 0)\right|^{p} d x\right)^{\frac{1}{p}}$

$+\left(\int_{\mathbb{R}^{N} \backslash B^{T}}\left|a_{3}(x)+b_{2}\right| g_{2}(x, u(x))\left|+b_{1} \int_{\mathbb{R}^{N}}\right| h_{2}(x, y,(Q u)(y)) d y||^{p} d x\right)^{\frac{1}{p}}$

$\leq\left(\int_{\mathbb{R}^{N} \backslash B^{T}}|f(x)|^{p} d x\right)^{\frac{1}{p}}+l\left(\int_{\mathbb{R}^{N} \backslash B^{T}}|u(x)|^{p} d x\right)^{\frac{1}{p}}+\left(\int_{\mathbb{R}^{N} \backslash B^{T}}\left|g_{1}(x, 0)\right|^{p} d x\right)^{\frac{1}{p}}$

$+\left(\int_{\mathbb{R}^{N} \backslash B^{T}}\left|a_{3}(x)\right|^{p} d x\right)^{\frac{1}{p}}+b_{2} l\left(\int_{\mathbb{R}^{N} \backslash B^{T}}|u(x)|^{p} d x\right)^{\frac{1}{p}}+b_{2}\left(\int_{\mathbb{R}^{N} \backslash B^{T}}\left|g_{2}(x, 0)\right|^{p} d x\right)^{\frac{1}{p}}$

$+$

$b_{1}\left(\int_{\mathbb{R}^{N} \backslash B^{T}}\left|\left(\int_{\mathbb{R}^{N}}|k(x, y)| \times\left[a_{4}(y)+b|(Q u)(y)|\right] d y\right)\right|^{p} d x\right)^{\frac{1}{p}}$

$\leq\left(\int_{\mathbb{R}^{N} \backslash B^{T}}|f(x)|^{p} d x\right)^{\frac{1}{p}}+l\left(\int_{\mathbb{R}^{N} \backslash B^{T}}|u(x)|^{p} d x\right)^{\frac{1}{p}}+\left(\int_{\mathbb{R}^{N} \backslash B^{T}}\left|g_{1}(x, 0)\right|^{p} d x\right)^{\frac{1}{p}}$

$+\left(\int_{\mathbb{R}^{N} \backslash B^{T}}\left|a_{3}(x)\right|^{p} d x\right)^{\frac{1}{p}}+b_{2} l\left(\int_{\mathbb{R}^{N} \backslash B^{T}}|u(x)|^{p} d x\right)^{\frac{1}{p}}$

$+b_{2}\left(\int_{\mathbb{R}^{N} \backslash B^{T}}\left|g_{2}(x, 0)\right|^{p} d x\right)^{\frac{1}{p}}+b_{1}\left(\int_{\mathbb{R}^{N} \backslash B^{T}}\left(\int_{\mathbb{R}^{N}}|k(x, y)|^{q}\left|a_{4}(y)\right|^{q} d y\right)^{\frac{p}{q}} d x\right)^{\frac{1}{p}}$ 


$$
+\quad b b_{1}\left(\int_{\mathbb{R}^{N} \backslash B^{T}}\left(\int_{\mathbb{R}^{N}}|k(x, y)|^{q}|(Q u)(y)|^{q} d y\right)^{\frac{p}{q}} d x\right)^{\frac{1}{p}}
$$

$\leq\|f\|_{L^{p}\left(\mathbb{R}^{N} \backslash B^{T}\right)}+l\|u\|_{L^{p}\left(\mathbb{R}_{N} \backslash B^{T}\right)}+\left\|g_{1}(., 0)\right\|_{L^{p}\left(\mathbb{R}_{N} \backslash B^{T}\right)}$

$+\left\|a_{3}\right\|_{L^{p}\left(\mathbb{R}^{N} \backslash B^{T}\right)}+b_{2} l\|u\|_{L^{p}\left(\mathbb{R}^{N} \backslash B^{T}\right)}+b_{2}\left\|g_{2}(., 0)\right\|_{L^{p}\left(\mathbb{R}^{N} \backslash B^{T}\right)}$

$+b_{1}$

$\left\|f^{*}\right\|_{L^{q}\left(\mathbb{R}^{N}\right)} \cdot\left\|f_{1}\right\|_{L^{p}\left(\mathbb{R}^{N} \backslash B^{T}\right)} \cdot\left(\left\|a_{4}\right\|_{L^{p}\left(\mathbb{R}^{N} \backslash B^{T}\right)}+b \psi(\|u\|)_{L^{p}\left(\mathbb{R}^{N}\right)}\right)$.

Also we have $\|f\|_{L^{p}\left(\mathbb{R}^{N} \backslash B^{T}\right)},\left\|g_{i}(., 0)\right\|_{L^{p}\left(\mathbb{R}^{N} \backslash B^{T}\right)}$,

$\left\|f_{1}\right\|_{L^{p}\left(\mathbb{R}^{N} \backslash B^{T}\right)},\left\|a_{3}\right\|_{L^{p}\left(\mathbb{R}^{N} \backslash B^{T}\right)} \rightarrow 0$

as $T \rightarrow \infty$ where $i=1,2$

and hence we obtain that

$$
d(F X) \leq l\left(b_{2}+1\right) d(X) .
$$

Consequentially we infer from equation $-13,-16$

$$
w_{0}(F X) \leq l\left(b_{2}+1\right) w_{0}(X),
$$

so, the operator $F$ satisfies all conditions of Darbo fixed point theorem, which enables us to deduce that $F$ has at least one solution inL $L^{p}\left(\mathbb{R}^{N}\right)$. Thus the proof is finished.

Next, we will need the following theorem that help us in a coming example.

\section{Theorem 3.2 [4]}

Let $\Omega \subseteq \mathbb{R}^{N}$ be a measure space and suppose $k: \Omega \times \Omega \rightarrow \mathbb{R}$ is a measurable function for which there is constant $C>0$ such that

$$
\int_{I}|k(x, y)| d x \leq C \quad \text { a.e. } y \in \Omega
$$

and

$$
\int_{I}|k(x, y)| d y \leq C \quad \text { a.e. } x \in \Omega \text {. }
$$

If $K: L^{p}(\Omega)$

$\rightarrow L^{p}(\Omega)$ is defined by

$$
(K f)(x)=\int_{\Omega} f(y) d y
$$

then $K$ is a bounded and continuous operator and $\|K\|_{1} \leq C$. 
Example: consider the integral equation

$\left(\mathrm{y}_{2} \frac{}{\left.1+y_{1}^{2}+2 e^{-|u(x)|} u(x)\right) d x}\right.$,

where

$$
x=\left(x_{1}, x_{2}\right) \in \mathbb{R}^{2},
$$

and $\|x\|$ is the Euclidean norm. We study the solvability of this integral equation in the space $L^{p}\left(\mathbb{R}^{2}\right)$ for $p, q>2$. Let $f(x)=e^{-x^{2}}, g_{1}(x, u(x))=\frac{\sin u}{\|x\|+4}$,

$h_{2}(x, y,(Q u)(y))=\frac{e^{-\left(\left|x_{1}\right|+\left|y_{1}\right|\right)}}{\left(\left|x_{2}\right|+3\right)^{2}\left(\left|y_{2}\right|+2\right)^{2}}\left(\frac{y_{2}}{1+y_{1}^{2}}+2 e^{-|u(x)|} u(x)\right)$,

$a(x, y)=e^{-x^{2}}+\frac{\sin u}{\|x\|+4}$ with $b_{1}=\frac{1}{8}, a_{3}(x)=e^{-x^{2}}$ where $a_{3} \in L^{p}\left(\mathbb{R}^{2}\right)$ such that $b_{2}=1, g_{2}(x, u(x))=\frac{\sin u}{\|x\|+4}$.

Hence the norm

$$
\|f\|_{L^{p}\left(\mathbb{R}^{2}\right)}=\left(\frac{\pi}{p}\right)^{\frac{1}{p}}
$$

Next the functions $g_{i}(x, u(x)), i=1,2$ satisfy the assumption(ii) with $a_{i}(x)=\frac{1}{\|x\|+4}, l=\frac{1}{4}$, indeed

$$
\begin{aligned}
\left|g_{i}(x, u)-g_{i}(y, v)\right| & =\left|\frac{\sin u}{\|x\|+4}-\frac{\sin v}{\|y\|+4}\right| \\
& \leq\left|\frac{1}{\|x\|+4}-\frac{1}{\|y\|+4}\right||\sin u|+\frac{1}{\|y\|+4}|\sin u-\sin v| \\
& \leq\left|\frac{1}{\|x\|+4}-\frac{1}{\|y\|+4}\right|+\frac{1}{4}|u-v| \\
& =\left|a_{i}(x)-a_{i}(y)\right|+l|u-v|
\end{aligned}
$$

where $a_{i}(x) \in L_{p}\left(\mathbb{R}^{2}\right)$ with norm

$$
\left\|a_{i}\right\|_{L^{p\left(\mathbb{R}^{2}\right)}}=\left(\frac{4 \pi(2)^{1-p}}{(p-1)(p-2)}\right)^{\frac{1}{p}}
$$

where $a_{4}=\frac{y_{2}}{1+y_{1}^{2}}$, with $\left\|a_{4}\right\|_{L^{p}\left(\mathbb{R}^{2}\right)}=0$, also

$$
k(x, y)=\frac{e^{-\left(\left|x_{1}\right|+\left|y_{1}\right|\right)}}{\left(\left|x_{2}\right|+3\right)^{2}\left(\left|y_{2}\right|+2\right)^{2}},
$$

$f^{*}(x)=\frac{e^{-\left|x_{1}\right|}}{\left(\left|x_{2}\right|+3\right)^{2}}, \quad f_{1}(x)=f_{2}(x)=\frac{e^{-\left(\left|x_{1}\right|\right)}}{\left(\left|x_{2}\right|+2\right)^{2}} \quad$ we see that $f_{1}, f_{2} \in L_{p\left(\mathbb{R}^{2}\right)}, f^{*} \in L_{q\left(\mathbb{R}^{2}\right)}$. Also we have

$$
\int_{\mathbb{R}^{2}}|k(x, y)| d x=\int_{-\infty}^{\infty} \int_{-\infty}^{\infty} \frac{e^{-\left(\left|x_{1}\right|+\left|y_{1}\right|\right)}}{\left(\left|x_{2}\right|+3\right)^{2}\left(\left|y_{2}\right|+2\right)^{2}} d x_{1} d x_{2} \leq \frac{1}{3}
$$

$$
\int_{\mathbb{R}^{2}}|k(x, y)| d y=\int_{-\infty}^{\infty} \int_{-\infty}^{\infty} \frac{e^{-\left(\left|x_{1}\right|+\left|y_{1}\right|\right)}}{\left(\left|x_{2}\right|+3\right)^{2}\left(\left|y_{2}\right|+2\right)^{2}} d y_{1} d y_{2} \leq \frac{2}{9}
$$


and thus from the theorem $\|K\|_{1} \leq \frac{1}{3}$ furthermore $\left.b=2, Q(u)(x)=e^{-|u(x)|} u(x)\right)$ satisfies the assumption with $\psi(t)=t$. Finally, the inequality from assumption (vi) has the form

$$
\begin{aligned}
& \|\quad f\|_{L_{p}\left(\mathbb{R}^{2}\right)}+l r_{0}+\left\|g_{1}(x, 0)\right\|_{L_{p}\left(\mathbb{R}^{2}\right)}+\left\|a_{3}\right\|_{L_{p}\left(\mathbb{R}^{2}\right)}+b_{2} l r_{0} \\
& +\quad b_{2}\left\|g_{2}(x, 0)\right\|_{L_{p}\left(\mathbb{R}^{2}\right)}+b_{1}\|K\|_{1}\left\|a_{4}\right\|_{L_{p}\left(\mathbb{R}^{2}\right)}+b b_{1}\|K\|_{1} \psi\left(r_{0}\right)
\end{aligned}
$$

$\leq r_{0}$

$$
2\left(\frac{\pi}{p}\right)^{\frac{1}{p}}+\frac{1}{2} r_{0}+\left(\frac{1}{4}\right)\left(\frac{1}{3}\right) r_{0} \leq r_{0}
$$

Thus, for the number $r_{0}=\left(\frac{24}{5}\right)$

$\times\left(\frac{\pi}{p}\right)^{\frac{1}{p}}$. Hence all the assumptions are satisfied and so, Eq.(3.4) has at least one solution in $L^{p}\left(\mathbb{R}^{2}\right)$.

\section{References}

[1] A. Aghajani, D. O'Regan and A.S. Haghighi, Measure of noncompactness on $L^{p}\left(\mathbb{R}^{N}\right)$ and applications, $C U B O$. March. 17(1) (2015), 85-97. https://doi.org/10.4067/S0719-06462015000100007

[2] A. A. El-Bary, M. A. Darwish, W. G. El-sayed, on an existence theorem for Uryshon integral equation via measure of noncompactness, Math. sci. Res. J. 6(9)(2002), 441- 448.

[3] A. Granas and J. Dugundji, Fixed point theory, Springer - Verlag, New York, 2003.

[4] B. Folland, Real Analysis, A Wiley-Interscience PUBLICATION, 1999.

[5] C. Corduneanu, Integral equations and applications, Cambridge: Cambridge University Press, 1991. https://doi.org/10.1017/CBO9780511569395

[6] D. Franco. G. infante and D. O'Regan, Positive and nontrivial solutions for the Urysohn integral equation, Acta Math. sin. (Engl. ser). 22(6)(2006), 1745- 1750. https://doi.org/10.1007/s10114-005-0782-3

[7] G. Darbo, Punti untiti in transformazioni a condominio noncompatto, Rend. Sem. Mat. Univ. Padora. 24 (1955), 84-92.

[8] H. Brezis, Functional Analysis, Sobolev Spaces and Partial Differential Equations, Springer New York Dordrecht Heidelberg London, 2011.

[9] H. Ha-Olsen, H. Holden, The Kolmogorov-Riesz compactness theorem,Expo. Math, 28(4)(2010), 385-394. https://doi.org/10.1016/j.exmath.2010.03.001

[10] H.K. Awad, M.A. Darwish and M.M.A. Metwali, On a Cubic Integral Equation of Urysohn Type with Linear Perturbation of Second Kind, J.Math. Appl. 41(2018), 29-38. 
[11] J. Banaś and W.G. El-Sayed, Solvability of functional and integral equations in some classes of integrable functions, Politechnika Rzeszowska, Rzeszow, 1993.

[12] J. Banas and K. Goebel, Measures of Noncompactness in Banach Spaces, Lect. Notes in Math. 60, M. Dekker, New york and Basel, 1980.

[13] J. Banaś, M. Lecko and W. G. El-Sayed, Existence theorems of some quadratic integral equations, J. Math. Anal. Appl. 222 (1998), 276-285.

https://doi.org/10.1006/jmaa.1998.5941

[14] J. Banaś and M. Pasalawska-Poludnik, Monotonic Solutions of Urysohn Integral equation on Unbounded interval, Comput. Math. Appl. 47(12)(2004), 1947- 1954.

https://doi.org/10.1016/j.camwa.2002.08.014

[15] J. Banaś, ,A. Chlebowicz, On integrable solutions of a nonlinear Volterra integral equation under Caratheodory conditions, Bull. Lond. Math. Soc. 41(6), 1073-1084, (2009).

[16] J. Banaś, Z. Knap, Measures of weak noncompactness and nonlinear integral equations of convolution type, J. Math. Anal. Appl. 146(2), 353-362, (1990).

[17] J. Banas, Z. Knap, Integrable solutions of a functional-integral equation, Revista Mat. Univ. Complutense de Madrid. 2(1989), 31-38.

[18] K. Deimling, Nonlinear functional analysis. Berlin. Springer-Verlag, 1985. https://doi.org/10.1007/978-3-662-00547-7

[19] K. Goebel and W. A. Kirk, Topics in metric fixed point theory, Cambridge University Press, 1990.

[20] L. N. Mishra, R. P. Agarwal, M. Sen, Solvability and asymptotic behavior for some nonlinear quadratic integral equation involving Erdélyi-Kober fractional integrals on the unbounded interval,Progress in Fractional Differentiation and Applications. 2(3) (2016), 153-168. URL: http://www.naturalspublishing.com/Article.asp?ArtcID=11601

[21] L.N. Mishra, H.M. Srivastava, M. Sen, On existence results for some nonlinear functional-integral equations in Banach algebra with applications, Int. J. Anal. Appl., 11(1)(2016), 1-10.

[22] L.N. Mishra, M. Sen, R.N. Mohapatra, On existence theorems for some generalized nonlinear functional-integral equations with applications, Filomat, 31(7)(2017), 2081-2091.

[23] L.N. Mishra, R.P. Agarwal, On existence theorems for some nonlinear functional-integral equations, Dynamic Systems and Applications, 25(2016), 303-320.

[24] L.N. Mishra, M. Sen, On the concept of existence and local attractivity of solutions for some quadratic Volterra integral equation of fractional order,Applied Mathematics and Computation.285(2016), 174-183. DOI: 10.1016/j.amc.2016.03.002

Recommendation: Based on above report, manuscript is

[25] M. Cichon and M. Metwali, On Monotonic Integrable Solutions for Quadratic Functional Integral Equations, Mediterr. J. Math. 10 (2013), 909-926.

https://doi.org/10.1007/s00009-012-0218-0 
[26] M. A. Darwish, On integral equations of Urysohn- Volterra type, Math. Comput. Appl. 136(1) (2003),93- 98. https://doi.org/10.1016/S0096-3003(02)00027-9

[27] M. A. Darwish, On aperturbed fuctional integral equation of Urysohn type, Comput. Appl. 218(2012), 8800- 8805. https://doi.org/10.1016/j.amc.2012.02.037

[28] M. A. Darwish, J. Henderson and D. O' Regan, existence and asymptotic solvability of solutions of aperturbed fractional fuctional- integral equation with linear modifications of the argument, Bull. Korean Math. Soc. 48(3)(2011), 539- 553 .

https://doi.org/10.4134/BKMS.2011.48.3.539

[29] M. A. Darwish, On solvability of some quadratic fuctional- integral equation in Banach algebra, J. Commun. Anal. Appl. 11(3-4) (2007), 441- 450.

[30] R. P. Agarwal and D. O'Regan, Infinite interval problems for differential, difference and integral equations., Dordrecht: Kluwer Academic Publishers, 2001.

[31] R. P. Agarwal, D. O'Regan, and P. J. Y. Wong, Positive solutions of differential, difference and integral equations, Dordrecht: Kluwer Academic Publishers, 1999.

[32] R. Agrawal, M. Meehan and D. O’ Regan, Fixed point theory and applications, Cambridge University Press, 2004.

[33] W. G. El-sayed, A. A. El-Bary and M. A. Darwish, solvability of Urysohn integral equation, Math. Comput. Appl.145(2003), 487- 493 .

https://doi.org/10.1016/S0096-3003(02)00504-0

[34] W. G. El-sayed and B. Rzepka, Nondecreasing solutionsof a quadratic integral equation of Urysohn type, Math. comput. Appl. 51(6- 7) (2006), 1065- 1074.

https://doi.org/10.1016/j.camwa.2005.08.033

[35] W. Al Sayed, M.A. Darwish, On the Existence of Solutions of a Perturbed Functional Integral Equation in the Space of Lebesgue Integrable Functions on $\mathbb{R}_{+}$, J. Math. Appl. 41(2018), 19-27.

\section{Data Availability (excluding Review articles)}

Applicable.

\section{Supplementary Materials}

Not applicable.

\section{Conflicts of Interest}

The authors declare that they have no competing interests.

\section{Funding Statement}

The research was self-sposored by the author.

\section{Acknowledgments}

The authors would like to express their thanks to the referee for his helpful comments and suggestions, which greatly improved the presentation of this paper. 